\title{
TERAPIA FÍSICA COMPLEXA NO LINFEDEMA EM PACIENTES APÓS CIRURGIA DE CÂNCER DE MAMA \\ - revisão sistemática・
}

\begin{abstract}
Marcela Augusta Moura de Roma*, Bianca Dantas Martins Pinheiro**, Daniele Costa Borges Souza***, Erika Pedreira da Fonseca****, Mansueto Gomes Neto*****, Helena França Correia dos Reis ${ }^{* * * * * *}$

Autor correspondente: Marcela Ausgusta Moura de Roma - marcelaroma@outlook.com

* Fisioterapeuta graduada pela Universidade Federal da Bahia. Residente em fisioterapia Hospitalar com Ênfase em Terapia Intensiva - Universidade Salvador (UNIFACS) - Hospital da Cidade/Hospital Geral Roberto Santos.

** Fisioterapeuta graduada pela Universidade Federal da Bahia.

*** Fisioterapeuta graduada pela Universidade Federal da Bahia. Fisioterapeuta do Hospital Ernesto Simões Filho - Unidade de Terapia Intensiva. Mestranda da Pós graduação em Medicina e Saúde da Faculdade de Medicina da Bahia - Universidade Federal da Bahia. Pós-graduanda em Fisioterapia Hospitalar pela Escola Bahiana de Medicina e Saúde Pública.

**** Fisioterapeuta. Mestre em Tecnologias em Saúde pela Escola Bahiana de Medicina e Saúde Pública. Pós-graduada em Reabilitação Neurofuncional - Faculdade Social da Bahia. Professora da Universidade Federal da Bahia.

***** Fisioterapeuta Mestre em Ciências da Reabilitação - Universidade Federal de Minas Gerais. Doutor em Medicina e Saúde - Universidade Federal da Bahia. Professor Adjunto I do departamento de Biofunção. Coordenador do curso de Fisioterapia e professor permanente do Programa de Pós-Graduação em Medicina e Saúde da UFBA.

****** Fisioterapeuta. Mestre em Medicina e Saúde Humana - Escola Bahiana de Medicina e Saúde Pública. Doutora em Medicina e Saúde Humana. Especialista em Fisioterapia Respiratória pela ASSOBRAFIR - Associação Brasileira de Fisioterapia Cardiorrespiratória e Fisioterapia em Terapia Intensiva. Professora adjunta da Universidade Federal da Bahia, Departamento de Biofunção, Curso de Fisioterapia.
\end{abstract}

\section{Resumo}

Objetivo: Reunir na literatura evidências sobre a efetividade da terapia física complexa no tratamento do linfedema em pacientes submetidas ao tratamento cirúrgico de câncer de mama. Método: Foram incluídos estudos de ensaios clínicos randomizados, publicados nas bases de dados indexadas no PubMed/Medline, SciELO, utilizando as principais palavras-chave: mastectomia, linfedema, cirurgia de câncer de mama, terapia física complexa, fisioterapia, terapia descongestiva completa e seus correlatos na língua inglesa. A qualidade metodológica dos artigos foi analisada de acordo com componentes individuais de avaliação segundo a cochrane collaboration. Resultados: Foram encontrados três estudos randomizados que comparam a terapia física complexa a outras formas de intervenção em pacientes com linfedema que foram submetidas à cirurgia da mama como forma de tratamento onde, dois artigos evidenciaram a efetividade da terapia física complexa na redução do linfedema. Conclusão: A terapia física complexa isolada parece ser a técnica mais efetiva para o tratamento do linfedema. Mais estudos randomizados e controlados são necessários para permitir a análise e comparação dos resultados.

Palavras-chave: Linfedema; Câncer de mama; Terapia física complexa. 


\title{
COMPLEX PHYSICAL THERAPY FOR LYMPHEDEMA IN POST BREAST CANCER SURGERY PATIENTS
}

\author{
-systematic review•
}

\begin{abstract}
Objective: To gather evidence in the literature on the effectiveness of complex physical therapy in the treatment of lymphedema in patients undergoing surgical treatment for breast cancer. Method: Studies with randomized clinical trials published in indexed databases PubMed / Medline, SciELO were included, using the main keywords: mastectomy lymphedema, breast cancer surgery, complex physical therapy, physiotherapy, complete decongestive therapy. The methodological quality of the articles was analyzed according to individual components assessment according to the Cochrane Collaboration. Result: Three randomized studies comparing the complex physical therapy to other forms of intervention in patients with lymphedema who underwent breast surgery as a treatment with two articles demonstrating the effectiveness of complex physical therapy in reducing lymphedema found. Conclusion: The complex physical therapy alone appears to be the most effective technique for the treatment of lymphedema. More randomized controlled trials are needed to enable the analysis and comparison of results.
\end{abstract}

Keywords: Lymphedema; Breast cancer; Complex physical therapy.

\section{INTRODUÇÃO}

Entre a população feminina, o câncer (CA) de mama possui alta prevalência, sendo considerado como um problema de saúde pública tanto em países desenvolvidos quanto em países em desenvolvimento. ${ }^{(1,2)}$ No Brasil é a principal causa de morte entre as mulheres, sobretudo devido ao diagnóstico e adoção de tratamentos tardios. ${ }^{(3,4)}$

Os avanços no tratamento do CA possibilitam uma nova perspectiva na qualidade de vida e sobrevida dos pacientes, mas ao mesmo tempo são responsáveis por diversas complicações precoces ou tardias. (5) Dentre as complicações físicas do momento pós-cirúrgico podemos destacar o linfedema, condição incapacitante crônica e sem cura, como uma das principais complicações decorrentes do tratamento do CA. ${ }^{(6-8)}$
O linfedema é uma manifestação clínica e patológica caracterizada pelo acúmulo de líquido com grande conteúdo proteico no interstício, resultante da insuficiência do sistema linfático em transportar pelos capilares e coletores o volume linfático que encontrasse em excesso. ${ }^{(9)}$

Sua ocorrência pode resultar em deformidade estética, perda de capacidade funcional, desconforto físico e sofrimento psicológico. ${ }^{(8)}$ Objetivando promover a reabsorção e a condução do líquido que se encontra acumulado para áreas normais e também para incentivar o desenvolvimento das vias colaterais de drenagem promovendo o controle do linfedema a longo prazo, a fisioterapia tem buscado formas de atuar no processo de tratamento. ${ }^{(10)}$

Dentre as opções de tratamento a Terapia Física Complexa (TFC) vem sendo preconizada para 
o tratamento do linfedema secundário ao CA de mama. Esta prática consiste na aplicação conjunta da drenagem linfática manual, cuidados com a pele, compressão com bandagem e exercícios miolifocinéticos em sua primeira fase e pela manutenção dos cuidados com o membro acometido em sua segunda fase. ${ }^{(8)}$

Sendo assim, esta revisão sistemática da literatura foi desenvolvida com o objetivo de reunir na literatura evidências sobre a efetividade desta terapia no tratamento do linfedema em pacientes submetidas ao tratamento cirúrgico de CA de mama.

\section{MATERIAL E MÉTODOS}

Foi realizada uma revisão sistemática nas bases de dados eletrônicas, nacionais e internacionais, MEDLINE via PubMed e Scielo, utilizando os seguintes descritores: mastectomia, linfedema, cirurgia de câncer de mama, terapia física comple$x a$, fisioterapia, terapia descongestiva completa, mastectomy, lymphedema, breast câncer surgery, complex physical therapy, physiotherapy, complete decongestive therapy, no período de março a maio de 2014. As combinações entre essas palavras foram realizadas em cada base de dados acima citadas utilizando os operadores booleanos $O R, A N D$, sem restrição de idioma e período de publicação. Referências encontradas nos estudos selecionados e que foram consideradas relevantes a esta revisão também foram incluídas.

Seguindo o protocolo pré-estabelecido, dois pesquisadores realizaram de forma independente a busca nas bases de dados. Os artigos encontrados foram, em um primeiro momento, submetidos à leitura critica dos títulos e de seus resumos, por cada um dos colaboradores, assim como a identificação daqueles que se encontravam em duplicidade.
Após reunião de consenso entre os colaboradores foram selecionados para a leitura integral os artigos que foram considerados relevantes ao objetivo desta revisão ou que geraram dúvida. Foram incluídos os estudos de ensaios clínicos randomizados, que descrevem os efeitos da TFC no tratamento do linfedema em pacientes que passaram por tratamento cirúrgico para o câncer de mama, sendo excluídos aqueles estudos que abordaram a validação de protocolo de atendimento e estudos que abordaram apenas as técnicas isoladas da TFC.

A avaliação da qualidade metodológica de componentes individuais para ensaio clínico randomizado deu-se de acordo com a Cochrane Collaboration. Os componentes analisados foram: tipos de randomização, sigilo de alocação, cegamento, analise por intenção de tratar, parada precoce por benefício, descrição seletiva do desfecho e escala validada, os quais podem ser classificados em três categorias: baixo risco de viés, alto risco de viés ou risco de viés incerto. ${ }^{(11)}$

\section{RESULTADOS}

Através da busca primária foram encontrados 148 artigos no Medline/Pubmed e 16 artigos no Scielo totalizando 164 artigos dos quais após leitura critica de títulos, resumos e analise das discordâncias entre os pesquisadores 26 artigos foram selecionados para a leitura integral dos textos. Após a leitura desses artigos, três estudos foram incluídos por preencherem os critérios de seleção para esta revisão sistemática (figura 1). Foi realizado busca manual das referências dos artigos selecionados, porém após análise das mesmas, nenhuma delas atendeu aos critérios de inclusão deste estudo. 


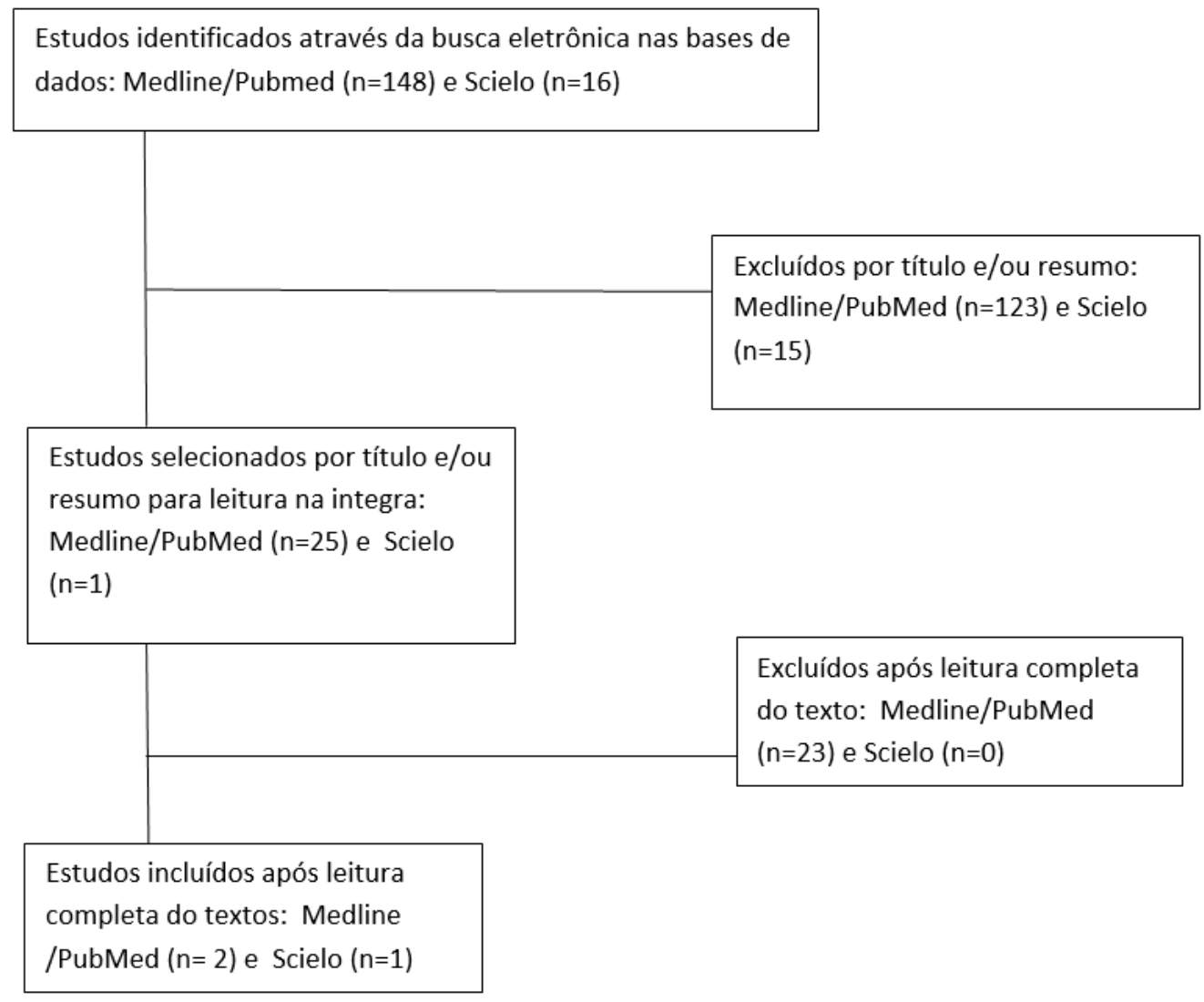

Figura 1 - Etapas de seleção dos artigos para revisão sistemática

Dos artigos selecionados a TFC (denominado nestes estudos de fisioterapia complexa descongestiva ou terapia complexa descongestiva) foi avaliada quanto a sua efetividade através de comparação com grupos que realizaram TFC associado a Compressão Pneumática Intermitente (CPI), ${ }^{(12)}$
TFC associado à ingestão de Triglicerídeos de Cadeia Média (TCM) ${ }^{(13)}$ e Fisioterapia Padrão (FP). ${ }^{(14)}$ As características dos estudos que preencheram os critérios de inclusão assim como as intervenções e resultados estão sumarizadas na tabela 1 . 
Tabela 1 - Descrição dos artigos incluídos

(continua)

\begin{tabular}{|c|c|c|c|c|c|}
\hline AUTOR & POPULAÇÃO & INTERVENÇÃO & CONTROLE & $\begin{array}{l}\text { DESFECHO/ } \\
\text { PARÂMETROS }\end{array}$ & REsultados \\
\hline $\begin{array}{l}\text { Haghighat } \\
\text { et al'2, } \\
2010\end{array}$ & $\begin{array}{l}112 \text { mulheres } \\
\text { submetidas à } \\
\text { cirurgia de CA } \\
\text { de mama }\end{array}$ & $\begin{array}{l}\text { Primeira fase } \\
\text { Grupo TFCM+ } \\
\text { CPI: } \\
\text { TFC e Manga } \\
\text { pneumática de } 4 \\
\text { câmaras com CPI } \\
40 \text { mmHg. } \\
\text { Fase de } \\
\text { manutenção } \\
\text { Compressão por } \\
\text { meia elástica } \\
\text { de 3/4 (dia); } \\
\text { curativos (noite) } \\
\text { cuidados com a } \\
\text { pele, Exercício, } \\
\text { Auto massagem } \\
\text { leve (1-2/dia) }\end{array}$ & $\begin{array}{l}\text { Primeira fase } \\
\text { Grupo TFC: } \\
\text { TFC (Cuidados } \\
\text { com a pele; } \\
\text { DLM; exercício; } \\
\text { bandagem } \\
\text { compressiva com } \\
\text { multicamadas) } \\
\text { Fase de } \\
\text { manutenção } \\
\text { Compressão por } \\
\text { meia elástica } \\
\text { de } 3 / 4 \text { (dia); } \\
\text { curativos (noite) } \\
\text { cuidados com a } \\
\text { pele, Exercício, } \\
\text { Auto massagem } \\
\text { leve (1-2/dia) }\end{array}$ & $\begin{array}{l}\text { Desfecho } \\
\text { primário: } \\
\text { redução do } \\
\text { linfedema após } \\
\text { primeira fase } \\
\text { do tratamento } \\
\text { e após } 3 \text { meses. } \\
\text { Parâmetro: } \\
\text { volumetria } \\
\text { Desfecho } \\
\text { secundário: } \\
\text { melhora de } \\
\text { sintomas } \\
\text { subjetivos }\end{array}$ & $\begin{array}{l}\text { Houve redução } \\
\text { significativa do } \\
\text { volume no final } \\
\text { da primeira fase } \\
\text { do tratamento } \\
\text { no grupo TFC } \\
\text { (43,1\% + 13,7\%) e } \\
\text { no Grupo TFC + } \\
\text { CPI (37,5\% + 14,4 } \\
\% \text { ) com diferença } \\
\text { estatisticamente } \\
\text { significativa. } \\
\text { (p=O,O36) } \\
\text { Após } 3 \text { meses de } \\
\text { seguimento houve } \\
\text { redução adicional } \\
\text { do volume nos } \\
\text { grupo TFC ( 16,9 } \\
\% \text { + 32,3 \%) e no } \\
\text { Grupo TFC + CPI } \\
\text { ( 7,5\% + 39,4 \%), } \\
\text { diferença não } \\
\text { estatisticamente } \\
\text { significativa. } \\
\text { (p=O,167) }\end{array}$ \\
\hline $\begin{array}{l}\text { Oliveira } \\
\text { et al'3., } \\
2008\end{array}$ & $\begin{array}{l}10 \text { mulheres } \\
\text { com idade } \\
\text { média de } 65,9 \\
\pm 10,4 \text { anos, } \\
\text { IMC de } 26,8 \\
\pm 3,0 \text { com } \\
\text { linfedema } \\
\text { de MS } \\
\text { homolateral à } \\
\text { cirurgia de CA } \\
\text { de mama }\end{array}$ & $\begin{array}{l}\text { Grupo TFC: } \\
\text { TFC (massagem } \\
\text { clássica, DLM } \\
\text { bandagem } \\
\text { compressiva e } \\
\text { cuidados com a } \\
\text { pele) }\end{array}$ & $\begin{array}{l}\text { Grupo TFC + } \\
\text { TCM: } \\
\text { TFC e ingestão } \\
\text { diária de TCM }\end{array}$ & $\begin{array}{l}\text { Desfecho } \\
\text { primário: } \\
\text { redução do } \\
\text { linfedema após } \\
\text { o final do tra- } \\
\text { tamento } \\
\text { Parâmetro: } \\
\text { perimetria e } \\
\text { volumetria, } \\
\text { pregas } \\
\text { cutâneas e } \\
\text { Bioimpedância } \\
\text { Desfecho } \\
\text { secundário: } \\
\text { melhora de } \\
\text { sintomas } \\
\text { subjetivos }\end{array}$ & $\begin{array}{l}\text { Houve redução } \\
\text { mais pronunciada } \\
\text { na perimetria do } \\
\text { grupo TFC+TCM } \\
\text { ( } \leq \leq 0,05) \\
\text { Houve redução } \\
\text { mais pronunciada } \\
\text { na volumetria do } \\
\text { grupo TFC+TCM } \\
\text { ( } \mathrm{p} \leq \text { O,O5) } \\
\text { Houve aumento } \\
\text { do volume do } \\
\text { membro após } \\
\text { tratamento no } \\
\text { grupo TFC }\end{array}$ \\
\hline
\end{tabular}




\begin{tabular}{|c|c|c|c|c|c|}
\hline AUTOR & População & INTERVENÇÃO & Controle & $\begin{array}{l}\text { DESFECHO/ } \\
\text { PARÂMETROS }\end{array}$ & Resultados \\
\hline $\begin{array}{l}\text { Didem } \\
\text { et al }{ }^{14} \text {., } \\
2005\end{array}$ & $\begin{array}{l}53 \text { mulheres } \\
\text { com idade } \\
\text { média de } 58 \\
\text { (31-76) anos, } \\
\text { submetidas à } \\
\text { cirurgia de CA } \\
\text { de mama }\end{array}$ & $\begin{array}{l}\text { Grupo TFC: } \\
\text { TFC (Cuidados } \\
\text { com a pele; } \\
\text { DLM; exercício; } \\
\text { bandagem } \\
\text { compressiva com } \\
\text { multicamadas) }\end{array}$ & $\begin{array}{l}\text { Grupo FP: } \\
\text { Bandagem, } \\
\text { elevação, } \\
\text { exercícios de } \\
\text { ombro e cabeça- } \\
\text { pescoço. }\end{array}$ & $\begin{array}{l}\text { Desfecho } \\
\text { primário: } \\
\text { redução do } \\
\text { linfedema após } \\
\text { o final do tra- } \\
\text { tamento } \\
\text { Parâmetro: } \\
\text { perimetria e } \\
\text { volumetria } \\
\text { Desfecho } \\
\text { secundário: } \\
\text { melhora da } \\
\text { mobilidade do } \\
\text { ombro }\end{array}$ & $\begin{array}{l}\text { Houve redução } \\
\text { percentual média } \\
\text { do edema mais } \\
\text { significativa para } \\
\text { o grupo TFC do } \\
\text { que para o grupo } \\
\text { FP }(55,7 \% \text { vs } 36 \% \text {, } \\
\text { respectivamente). } \\
\text { ( } p<0,005 \text { ) }\end{array}$ \\
\hline
\end{tabular}

TFC : terapia física complexa, CPI: compressão pneumática intermitente, TCM: triglicerídeos de cadeia média , FP: fisioterapia padrão

A avaliação da qualidade metodológica através da análise dos componentes individuais pelos cri- térios da Colaboração Cochrane ${ }^{(1)}$ estão descritos na quadro 1.

Quadro 1 - Analise da Qualidade metodológica

\begin{tabular}{|l|c|c|c|c|c|c|c|}
\hline \multicolumn{1}{|c|}{ ESTUDO } & RANDOMIZAÇÃo & $\begin{array}{c}\text { SIGILO DE } \\
\text { ALOCAÇÃo }\end{array}$ & CEGAMENTO & $\begin{array}{c}\text { ANALISE } \\
\text { POR } \\
\text { INTENÇÃo } \\
\text { DE TRATAR }\end{array}$ & $\begin{array}{c}\text { PARADA } \\
\text { PRECOCE } \\
\text { POR } \\
\text { BENEFICIO }\end{array}$ & $\begin{array}{c}\text { SELEÇÃo } \\
\text { DESCRITIVA } \\
\text { Do } \\
\text { DESFECHO }\end{array}$ & $\begin{array}{c}\text { EsCALA } \\
\text { VALIDADA }\end{array}$ \\
\hline $\begin{array}{l}\text { Haghighat } \\
\text { et al., } \\
\mathbf{2 0 1 0}\end{array}$ & Baixo risco & Baixo risco & Incerto & $\begin{array}{c}\text { Baixo } \\
\text { risco }\end{array}$ & Baixo risco & Baixo risco & $\begin{array}{c}\text { Baixo } \\
\text { risco }\end{array}$ \\
\hline $\begin{array}{l}\text { Oliveira et al, } \\
\mathbf{2 0 0 8}\end{array}$ & Incerto & Alto risco & $\begin{array}{c}\text { Baixo } \\
\text { risco }\end{array}$ & $\begin{array}{c}\text { Baixo } \\
\text { risco }\end{array}$ & Baixo risco & Baixo risco & $\begin{array}{c}\text { Baixo } \\
\text { risco }\end{array}$ \\
\hline $\begin{array}{l}\text { Didem et al., } \\
\mathbf{2 0 0 5}\end{array}$ & Baixo risco & Incerto & Incerto & $\begin{array}{c}\text { Baixo } \\
\text { risco }\end{array}$ & Baixo risco & Baixo risco & $\begin{array}{c}\text { Baixo } \\
\text { risco }\end{array}$ \\
\hline
\end{tabular}

O estudo de Haghighat et al $^{(12)}$ apresentou viés incerto para cegamento. No estudo de Oliveira et al, ${ }^{13)}$ houve viés incerto para randomização e alto risco de viés para sigilo de alocação e no estudo de
Didem et al ${ }^{(14)}$ foi avaliado viés incerto para sigilo de alocação e cegamento.

Os estudos avaliaram um total de 175 pacientes submetidas à cirurgia da mama e que passa- 
ram por algum tratamento adjuvante com radioterapia, ${ }^{(12-14)}$ quimioterapia ${ }^{(12-14)}$ e terapia endócrina. (14) No estudo de Haghighat et al ${ }^{(12)}$ foram incluídas mulheres que passaram por mastectomia radical modificada e cirurgia conservadora da mama. Oliveira et al ${ }^{\left({ }^{13)}\right)}$ incluíram mulheres com mastectomia radical modificada e quadrandectomia e Didem et al ${ }^{(14)}$ incluíram pacientes com mastectomia radical, mastectomia radical modificada e lumpectomia.

Para avaliação do edema do membro superior os autores utilizaram como métodos de mensuração a volumetria por deslocamento de água e a perimetria, no entanto empregando diferentes pontos anatômicos para a realização das medidas. Somente os estudos de Haghighat et $\mathrm{al}^{(12)}$ e Didem et al(14) determinaram o ponto de corte considerado para definir o linfedema. O primeiro considerou um aumento maior igual a $10 \%$ no volume do braço afetado em comparação com o braço contralateral para inclusão dos pacientes enquanto o segundo adotou como ponto de corte a diferença de até $2 \mathrm{~cm}$ (linfedema leve) e entre 2 a $5 \mathrm{~cm}$ (linfedema moderado) na circunferência dos membros.

Os ensaios clínicos não foram homogêneos quanto ao tempo de administração da TFC, e tempo de seguimento das pacientes. No estudo de $\mathrm{Ha}$ ghighat et al, ${ }^{(12)} 112$ mulheres foram randomizadas em dois grupos de igual quantidade de indivíduos: Grupo TFC, por fins de estudo considerado como grupo padrão ou controle, que recebeu TFC durante cinco dias por 10 a 15 sessões e o grupo TFC associado a compressão pneumática intermitente que recebeu TFC associada a CPI por igual período na primeira fase do tratamento. A fase de manutenção da TFC foi empregada em ambos os grupos até três meses do final da primeira fase por meio de atividades educativas que deveriam ser realizados no domicilio da paciente. Assim a redução do linfedema foi avaliado após o final da primeira fase e a redução adicional após três meses.

Didem et al, (14) realizaram um estudo em 53 muIheres divididas aleatoriamente em dois grupos para receber TFC $(n=27)$ ou FP $(n=26)$ e Oliveira et $a^{\left({ }^{(13)}\right.}$ em seu estudo avaliaram 10 mulheres divi- didas em grupos que receberam TFC $(n=5)$ e TFC associada ingestão diária de TCM $(n=5)$. Ambos os estudos realizaram as intervenções por três dias durante quatro semanas avaliando os achados dos tratamentos somente no momento imediato ao fim do tratamento.

Os componentes da TFC empregados nos estudos divergiram. Haghighat et al ${ }^{(12)}$ e Didem et al,(14) utilizaram a drenagem linfática manual (técnica de Vodder e modificação da técnica de Földi e StroBenneother, respectivamente), bandagem compressiva, exercícios e cuidados com a pele enquanto, Oliveira et al ${ }^{(13)}$ utilizaram a massagem clássica, drenagem linfática manual e bandagem compressiva.

Nos estudos de Haghighat et al ${ }^{(12)}$ e Didem et al, ${ }^{(14)}$ ambas as terapias empregadas nos grupos de intervenção apresentaram resultados estatisticamente significativos para a redução do linfedema, porém ambos referem que maior redução foi obtida através da utilização da TFC isolada do que quando associada a compressão pneumática intermitente ou quando esta foi comparada a fisioterapia padrão. No estudo de Oliveira et $a^{\left({ }^{(3)}\right)}$ a intervenção que associou a TFC à ingestão de TCM, foi a que apresentou desfecho favorável a redução do linfedema e a TFC sozinha, apresentou efeito negativo refletindo em aumento do volume médio entre membro acometido e sadio dos indivíduos deste grupo.

Em relação à avaliação de medidas das pregas cutâneas tricipital e bicipital, por adipômetro e da quantidade de água corporal total, por meio do exame de bioimpedância elétrica de múltipla frequência, foram analisados apenas no estudo de Oliveira et al, ${ }^{(13)}$ não sendo encontrada redução significativa das medidas de pregas cutâneas do membro acometido na comparação com os valores finais entre os grupos, assim como não houve diferenças significativas em comparação com a média de redução da quantidade de água corporal total entre os grupos após as intervenções.

Sensação subjetiva de dor, parestesia e sensação de peso foram avaliadas em dois estudos. ${ }^{(12,13)} \mathrm{No}$ 
estudo de Haghighat et a $\mathrm{a}^{(12)}$ o escore dos sintomas diminui para ambos métodos durante o período do estudo e a somente a sensação de peso, durante a primeira fase, foi estatisticamente significativa no grupo TFC quando comparado com o outro grupo. Em Oliveira et al ${ }^{(13)}$ quando comparados os valores iniciais e finais houve diferença estatisticamente significativa na diminuição dos graus de sensação de desconforto dentro de cada grupo e a diminuição da sensação de peso no braço foi significativa apenas no grupo TFC associado a ingestão de TCM ao final da intervenção e entre os dois grupos não houve diferença das demais sensações analisadas.

Somente o estudo de Didem et $a^{\left({ }^{(14)}\right.}$ realizou a medida de amplitude de movimento do ombro para os movimentos ativos de extensão-flexão, abdução-adução e rotação externa medidos por goniometria, encontrando aumento estatisticamente significativo para os movimentos de flexão e abdução do ombro em ambos os grupos de intervenção, mas que não foram significativos entre os grupos.

\section{DISCUSSÃO}

O linfedema é uma complicação de característica crônica, comum aos pacientes tratados cirurgicamente para o câncer de mama devido aos danos produzidos no sistema linfático. Sua presença causa deformidade, restrição e incapacidade funcional e podem provocar um maior índice de estresse, depressão e sofrimento emocional. Para o adequado manejo do linfedema é fundamental que se busque formas de atuar sobre o sistema linfático favorecendo assim a diminuição da progressão e severidade do edema. ${ }^{(15)}$

Os resultados da presente revisão demonstraram que a TFC isolada, quando comparado a outros métodos, encontrou melhores resultados para a redução do linfedema em dois estudos ${ }^{(12,14)}$ e somente em um estudo houve resultado contrário. ${ }^{(13)}$

Em relação à qualidade metodológica dos estudos selecionados o de Haghighat et al ${ }^{(12)}$ apresentou viés para cegamento, Oliveira et al ${ }^{\left({ }^{(13)}\right.}$ apresenta- ram vieses para randomização e sigilo de alocação enquanto Didem et al ${ }^{\left({ }^{(4)}\right)}$ apresentou viés para sigilo de alocação e cegamento. Vieses de randomização e sigilo de alocação referem-se à possibilidade de viés de seleção. ${ }^{(1)} A$ randomização quando realizado por métodos de geração de listas randômicas utilizando computador ou através de tabela de números randômicos promove o equilíbrio entre os grupos estudados tornando-os semelhantes e assim possibilitando que os resultados encontrados após as intervenções sejam decorrentes apenas do tratamento realizado. ${ }^{(16)}$

Somente o estudo de Oliveira et al ${ }^{\left({ }^{(3)}\right)}$ descreveu como foi realizado cegamento dos participantes quanto a intervenção, desta forma limita-se que opiniões, quer sejam otimistas ou pessimistas, possam distorcer, sistematicamente, os aspectos e o relato do objetivo do tratamento, o que poderia reduzir, consequentemente, a confiança nos resultados do estudo. ${ }^{(16)}$

$\mathrm{O}$ estudo de Haghighat et $\mathrm{a}^{(12)}$ ao considerar a TFC isolada demostrou que dentre as sensações e sintomas avaliados, apenas houve redução significativa para sensação de peso no membro acometido e, no estudo de Oliveira et al(13) somente o grupo que realizou a TFC associada a TCM foi o que apresentou resultado semelhante.

No estudo em que a TFC foi associado a CPI (12) questionou-se o possível aumento do efeito da TFC provocado por esta combinação, o que não foi encontrado neste estudo. Porém outros estudos ainda mostram-se contraditórios quanto a este fato pois em alguns este maior efeito foi encontrado para a redução do linfedema de membro superior $^{(17,18)}$ enquanto outro não encontrou tal efeito quando foi aplicado em pacientes que apresentaram linfedema em membro inferior. ${ }^{(19)}$ Uma recente revisão sistemática corrobora com os achados acima citados, concluindo que são necessárias maiores evidências para dar suporte ao uso da CPI no tratamento do linfedema. ${ }^{(20)}$

No estudo de Oliveira et al ${ }^{(13)}$ a associação da TFC à ingestão da TCM demostrou ser efetiva na redução tanto do volume quanto da perimetria do 
membro acometido enquanto, que na TFC isolada houve aumento do linfedema. Os autores do referido estudo questionam se o emprego de óleo vegetal de milho como fonte de gordura contendo em sua maior parte TCL que são absorvidos pelo sistema linfático, fato que ocorre em menor quantidade quando se faz o uso de TCM, fornecido como forma de placebo, aos indivíduos que faziam parte do grupo TFC, tenha contribuído para a não redução do linfedema. ${ }^{(13)}$

Em relação à mobilidade do ombro o estudo de Didem et al ${ }^{(14)}$ realizou tal medida, encontrando aumento estatisticamente significativo tanto ao realizar a TFC isolada quanto através da FP não demostrando superioridade da TFC na melhora da amplitude de movimento do ombro para os movimentos de flexão e abdução.

Há interesse crescente sobre a qualidade de vida dos pacientes com linfedema, porém, ainda se observa que os estudos tem centralizado seu foco na redução do linfedema, esquecendo que o paciente experiência uma variedade de dificuldades físicas e psicológicas, incluindo a depressão, imagem corporal negativa e dor, que provavelmente podem ser beneficiados com o tratamento, mas que no entanto não são avaliados através de um abordagem clínica baseada na qualidade de vida. ${ }^{(21)}$

Nenhum dos estudos avaliou melhora da qualidade de vida dos indivíduos após o emprego das intervenções impossibilitando inferir se há alguma associação com o emprego da TFC. Apenas o estudo Haghighat et al ${ }^{(12)}$ avaliou efeitos da TFC por um tempo de seguimento de três meses em que a redução do edema apesar de existir, não foi estatisticamente significativa, necessitando que mais estudos sejam realizados com o intuito de investigar por maior tempo os efeitos sobre a manutenção e ganho adicional na redução do edema, uma vez este é um quadro crônico que acompanhará o indivíduo durante toda sua vida.

Para definir a presença do linfedema os estudos determinaram as medidas utilizando a diferença de volume ou a medidas de perimetria dos membros. Ambas as técnicas são utilizadas em diversos estu- dos, porém a medida por volumetria com deslocamento de água é a que apresenta melhor sensibilidade para detecção do linfedema e suas medidas. (22)

A técnica de mensuração do linfedema por bioimpedância foi utilizada somente em um estudo porém quando disponível é um método de diagnóstico que traz como vantagens a capacidade de distinguir se as alterações no volume são devido a atrofia muscular, ou a aumentos na massa magra, gordura corporal disponível ou devido a melhora na drenagem da linfa. Sendo um método mais sensível para identificação de pequenas alterações no fluido intersticial e linfático, importante quando nem a perimetria e volumetria forem capazes de identificar alterações linfáticas. ${ }^{(23)}$

\section{CONCLUSÃO}

Baseada na presente revisão, dois estudos mostraram que a terapia física complexa sozinha parece ser a técnica mais efetiva para o tratamento do linfedema em pacientes submetidas a cirurgia de câncer de mama. Este estudo apresenta algumas limitações. O número reduzido de publicações e a heterogeneidade na técnica utilizada impossibilitou a comparação entre os estudos e realização de metanálise. Desse modo, há a necessidade de estudos futuros metodologicamente adequados que permitam a análise e comparação dos resultados e uma conclusão mais consistente para recomendação ampla da técnica.

\section{REFERÊNCIAS}

1. Instituto Nacional de Câncer Ministério da Saúde (Brasil). Estimativa 2014: incidência de câncer no Brasil - Rio de Janeiro: Inca; 2014. [citado 2014 maio]. Disponível em: http://www.inca.gov.br/estimativa/2O14/ estimativa-24042014.pdf

2. Ferlay J, Shin HR, Bray F, Forman D, Mathers C, Parkin DM. Estimates of worldwide burden 
of cancer in 2008: GLOBOCAN 2008. Int. J. cancer. 2010;127(12):2893-2917.

3. Sclowitz ML, Menezes AMB, Gigante DP, Tessaro S. Condutas na prevenção secundária do câncer de mama e fatores associados. Rev. saúde pública. 2005;39(3):340-49.

4. Brasil. Ministério da Saúde. Controle do câncer de mama- Documento de consenso. Rev. bras. cancerol. 2004;50(2):77-90.

5. Tiezi DG. Cirurgia conservadora no câncer de mama. Rev. bras. ginecol. obstet. 2007;29 (8):428-34.

6. Rezende LF, Rocha AVR, Gomes CS. Avaliação dos fatores de risco no linfedema póstratamento de câncer de mama. J. vasc. Bras. 2010;9(4):233-38.

7. Norman SA, Localio AR, Potashnik SL, Simoes Torpey HA, Kallan MJ, Weber AL, et al. Lymphedema in breast câncer survivors: incidence, degree, time course, treatment and symptoms. J. clin. oncol. 2003;27(3):84-91.

8. The International Society of Lymphology. The Diagnosis and Treatment of Peripheral Lymphedema: 2013 Consensus Document of the International Society of Lymphology. Lymphology. 2013;46(1):1-11.

9. Rezende LF, Ramos FV, Ramos CD, Gurguel MSC. Avaliação das compensações linfáticas no pós-operatorio de câncer de mama com dissecção axilar através da linfocintilografia. J. vasc. bras 2008;7(4):370-74.

10. Luz ND, Lima ACG. Recursos fisioterapêuticos em linfedema pós-mastectomia: uma revisão de literatura. Fisioter. pesqui. 2011;24(1):191-200.

11. Carvalho APV, Silva V, Grande AJ. Avaliação do risco de viés de ensaios clínicos randomizados pela ferramenta da colaboração Cochrane. Diagn. tratamento 2013;18(1):38-44.

12. Haghighat S, Lotfi-Tokaldany M, Yunesian M, Akbari ME, Nazemi F, Weiss J. Comparing two treatment methods for post mastectomy lymphedema: complex decongestive therapyalone and in combination with intermittent pneumatic compression. Lymphology. 2010;43(1):25-33.

13. Oliveira J, Cesar TB. Influência da fisioterapia complexa descongestiva associada à ingestão de triglicerídeos de cadeia média no tratamento do linfedema de membro superior. Rev. bras. fisioter. 2008;12(1):31-36.

14. Didem K, Ufuk YS, Serdar S, Zümre A. The comparison of two diferente physiotherapy methods in treatment of lymphedema after breast surgery. Breast cancer res. Treat. 2005;93(1):49-54.

15. Gómez-Sadornil AM, Martín-Nogueras AM. Eficacia de la fisioterapia en el linfedema posmastectomía. Fisioterapia. 2013. http:// dx.doi.org/10.1016/j.ft.2013.11.001

16. HP, Avezum A. Princípios básicos e aplicações da cardiologia baseada em evidências Rev. bras. hipertens. 2009;16(1):48-51.

17. Rockson S, Miller L, Senie R, et al. American Cancer Society Lymphedema Workshop. Workgroup III: Diagnosis and management of lymphedema. Cancer. 1998;15;83(12 Suppl American):2882-5.

18. Szuba A, Achalu R, Rockson SG. Decongestive Lymphatic Therapy for Patients with Breast Carcinoma-Associated Lymphedema A Randomized, Prospective Study of a Role for Adjunctive Intermittent Pneumatic Compression. Cancer. 2002;95(11):2260-7.

19. Szolnoky G, Nagy N, Kovacs RK, et al: Complex decongestive physiotherapy decreases capillary fragility in lipedema. Lymphology. 2008;41(4):161-6.

2O. Rech JBS, Nóbrega L, Lemos A. Compressão Pneumática no Tratamento de Linfedema Pósmastectomia: Revisão Sistemática. Rev. bras. cancerol.2010;56(4):483-491.

21. Kim SJ, Yi CH, Kwon OY. Effect of complex decongestive therapy on edema and the quality of life in breast cancer patients with unilateral leymphedema. Lymphology. 2007; 4O(3):143-51.

22. Galland C, Auvert JF, Flahault A, Vayssairat M. Why and how post-mastectomy edema should be quantified in patients with breast cancer. Breast Cancer res. treat. 2002;75(1):87-9.

23. Godoy JM, Valente FM, Azoubel LM, Godoy MF. Evaluation of lymph drainage using bioelectrical impedance of the body. Phlebology. 2011;26(7):298-300. 